\title{
A genotypic ascertainment approach to refute the association of MYO1A variants with non-syndromic deafness
}

\author{
John Patton ${ }^{1,3}$, Carmen Brewer ${ }^{2}$, Wade Chien ${ }^{2}$, Jennifer J Johnston ${ }^{1}$, Andrew J Griffith ${ }^{2}$ \\ and Leslie G Biesecker ${ }^{\star}$
}

Variants in the unconventional myosin gene, MYO1A, have been reported to cause non-syndromic sensorineural hearing loss with a pattern of autosomal dominant inheritance. Others have challenged this association. We used a genotypic ascertainment study design to test the association of MYO1A variants with hearing loss. We evaluated MYO1A variants from a cohort of 951 individuals with exome sequencing who were not ascertained for hearing loss. Five individuals had one of two variants claimed to be associated with sensorineural hearing loss in a prior study and 33 individuals had one of 13 predicted deleterious variants. We obtained audiology evaluations for 12 individuals with these variants of interest. The hearing acuity of the participants was compared with age- and sex-matched controls and published age- and sex-specific reference ranges from a large population of otologically screened adults. None of the participants had bilateral sensorineural hearing loss of moderate or greater severity. These data do not support a causal relationship of variants in MYO1A to sensorineural hearing loss. We suggest that the genotypic ascertainment method is useful to objectively evaluate gene-phenotype associations.

European Journal of Human Genetics (2017) 25, 147-149; doi:10.1038/ejhg.2016.140; published online 19 October 2016

\section{INTRODUCTION}

Hearing impairment is common-one in two individuals develop an auditory deficit of greater than $25 \mathrm{~dB}$ HL by 80 years of age. ${ }^{1}$ Hearing loss is attributable to genetic and environmental factors. Currently, mutations in 33 genes have been associated with autosomal dominant non-syndromic hearing loss (ADNSHL, Hereditary Hearing Loss Homepage, URL: http://hereditaryhearingloss.org June 2015). One of the genes that has been claimed to be associated with ADNSHL is MYO1A. ${ }^{2}$ The authors of that report wrote 'MYO1A is the first myosin I family member found to be linked to human hereditary deafness...' and 'Finally, the identification of at least six different mutations suggests that this gene may be a significant contributor to autosomal dominant cases.' In North America, this would be considered an assertion of pathogenicity for these variants. However, it is unclear whether this is valid., ${ }^{3,4}$ We set out to test this hypothesis using a distinct approach.

Human genetic research studies commonly apply a phenotype-first paradigm with individuals (and matched controls) ascertained for a phenotype who subsequently undergo molecular characterization. This can lead to erroneous associations. ${ }^{5}$ To address the challenges and limitations of the phenotype ascertainment paradigm, ${ }^{6,7}$ we applied a novel genotype-ascertainment approach to genetic association. In this approach, exome or genome sequencing of a broadly ascertained cohort is performed first. Genotype results are then used to select a subset of individuals and controls for targeted phenotype characterization. We applied this to individuals who were not ascertained for, nor excluded for, hearing loss, for whom exome data were available.
We selected individuals who had MYO1A variants and then performed post hoc phenotyping for hearing loss. In this way, we hoped to reduce one of the components of ascertainment bias and test the association of variants in this gene with hearing status.

\section{MATERIALS AND METHODS}

Participant enrollment

The participants were enrolled through the ClinSeq project. ${ }^{8}$ They ranged in age from 45 to 65 years at enrollment and were selected for a range of atherosclerosis phenotypes, but not for personal or family histories of hearing impairment, nor were individuals with hearing loss excluded. The ClinSeq study has been reviewed and approved by the NHGRI IRB and included written informed consent for the primary study. Blood samples were obtained for exome sequencing. Massively parallel sequencing procedures have been described. ${ }^{9}$ Twelve individuals with variants of interest (see below) in MYO1A (cases) and twelve individuals without MYO1A variants (controls) were invited to the NIH to receive an audiology evaluation including measurement of pure-tone thresholds, tympanometry, and a hearing-focused history interview. The controls were selected by identifying the closest age- and sex-matched individual within the ClinSeq data set to the cases who agreed to participate. Five of these participants (three with variants of interest and two controls) opted to submit an outside audiology evaluation and participated in the history interview over the phone. Informed consent for this substudy was obtained by telephone conversation. The mean age of cases was 61.8 years

\footnotetext{
${ }^{1}$ Medical Genomics and Metabolic Genetics Branch, National Human Genome Research Institute, National Institutes of Health, Bethesda, MD, USA; ${ }^{2}$ Otolaryngology Branch, National Institute on Deafness and other Communication Disorders, National Institutes of Health, Bethesda, MD, USA

${ }^{3}$ Current address: Des Moines University College of Osteopathic Medicine, Des Moines, IA, USA.

${ }^{*}$ Correspondence: Dr LG Biesecker, Chief, Medical Genomics and Metabolic Genetics Branch, National Human Genome Research Institute, National Institutes of Health, 50 South Drive Room 5140, Bethesda, MD 20892-4472 USA. Tel: +1301 402 2041; Fax: +1301 480 0353; E-mail: lesb@mail.nih.gov
}

Received 23 December 2015; revised 4 September 2016; accepted 13 September 2016; published online 19 October 2016 
and the mean age of controls was 62.5 years. Seven (58\%) of the 12 case-control pairs were male and five (42\%) were female.

\section{Variant analysis}

Exome data were generated from 951 participants enrolled in ClinSeq, who had among them 2456493 unique variants. The data were filtered for quality and frequency. A Most Probable Genotype score ${ }^{10}$ of $\geq 10$ and a minor allele frequency of $\leq 0.01$ were set as threshold values for inclusion. Participants with variants in MYO1A reported as pathogenic and variants predicted to be pathogenic were invited to participate in the study. Predicted pathogenicity was evaluated by a Combined Annotation Dependent Depletion ${ }^{11}$ score of $\geq 20$. Mutation nomenclature conforms with HGVS recommendations and is referenced to NM_005379.3 or NC_000012.12. Exome data have been deposited into dbGAP (phs000971.v1.p1 NHGRI ClinSeq) and the variants have been submitted to ClinVar.

\section{Audiology analysis}

Hearing thresholds of each participant were compared with the 95th centile age- and sex-based reference ranges from the ISO 7029 (International Standards Organization) cohort of otologically screened adults. ${ }^{12}$ The better-hearing ear was determined based on the fourfrequency $(500,1000,2000$, and $4000 \mathrm{~Hz})$ pure-tone average or, in the case of equal pure tone averages, the $4000 \mathrm{~Hz}$ threshold was used to select the better-hearing ear. Air conduction thresholds were used unless there was a conductive (middle ear) hearing loss (bone conduction thresholds better than air conduction thresholds by $>10 \mathrm{~dB}$ ). In this case, bone conduction thresholds were used to represent sensory hearing acuity. The clinical degree of hearing loss was categorized as follows: $\leq 20 \mathrm{~dB} \mathrm{HL}=$ normal, $>20-40 \mathrm{~dB} \mathrm{HL}=$ mild, $>40-70 \mathrm{~dB} \mathrm{HL}=$ moderate, $>70-95 \mathrm{~dB} \mathrm{HL}=$ severe, and $>95 \mathrm{~dB} \mathrm{HL}=$ profound. ${ }^{13}$

\section{RESULTS}

There were 40 distinct variants in MYO1A among 951 participants (See Supplementary Table 1). Two variants were excluded because they had a MAF of 0.0799 and 0.0374 , which failed our frequency filter. We invited all individuals harboring a variant described by Donaudy et al as associated with hearing impairment and all those with combined annotation dependent depletion scores $\geq 20$ to participate.

Twelve individuals, heterozygous for one each of six variants of interest, participated in the study (Supplementary Table 1) and were matched with 12 controls. Auditory testing results for the 24 tested individuals (12 cases and 12 controls) are shown in Supplementary Table 2.

Five of the 12 participants had variants previously claimed by Donaudy et al to cause ADNSHL. Four of these five had c. $277 \mathrm{C}>\mathrm{T}$ p. $\left(\operatorname{Arg} 93^{*}\right)$. Of the four with p. $($ Arg93*), two (participants 185517 and 136069) had hearing acuity within the ISO age- and sex-based 95th centile ranges. The other two (160088 and 129395) had mild lowfrequency hearing loss thresholds that were outside the 95th centile. Of the five with previously reported variants, one had the c.2021G > A p. (Gly674Asp) variant. He was a 66-year-old man (132986) with hearing within ISO norms.

Seven additional individuals had variants predicted to be deleterious based on combined annotation dependent depletion scores $\geq 20$. A c. $1011+2 T>G$ predicted loss-of-function variant was found in a 63 year-old woman (153417) whose hearing was within ISO norms. The c. $640+1 \mathrm{G}>\mathrm{C}$ predicted loss-of-function variant was found in a 62 year-old woman (125959) whose hearing was normal. The c.235G $>\mathrm{T}$ p.(Ala79Ser) missense variant was identified in a 71-year-old man
(150934) with hearing within the ISO norms. Four individuals had c.1882C > G p.(Arg628Gly). All four (169143, 166357, 128503, and 153663) had thresholds within the ISO 95th centiles bilaterally.

The pure-tone averages of the 12 individuals described above were compared with the pure-tone averages of the controls. A multivariate linear regression showed no significant association of hearing loss and the presence of a MYO1A variant, controlling for age and gender. The presence of a MYO1A variant yielded a coefficient of $4.342 \pm 2.899$, $P=0.151$. Age had a coefficient of $0.577 \pm 0.300, P=0.070$. Gender had a coefficient of $3.665 \pm 3.016, P=0.245$. The four-frequency puretone average for participants with putative pathogenic variants was $18.75 \mathrm{~dB} H L \pm 7.19$. Controls from the cohort had a four-frequency pure-tone average of $14.79 \mathrm{~dB} H L \pm 7.01$, and the age- and sexmatched ISO 7029 controls had a four-frequency pure-tone average of $12.39 \mathrm{~dB} H L \pm 2.93$. The pure-tone averages of participants with variants were not different from those of cohort controls (paired $t$ test, $P=0.163$ ) but did differ from those of ISO 7029 controls $(P=0.0156)$, however, a difference of $6 \mathrm{~dB}$ is not clinically significant.

The exomes of the participants described here were also screened for reported or predicted loss-of-function variants in the 99 genes associated with non-syndromic deafness in the hereditary hearing loss homepage (http://hereditaryhearingloss.org/ accessed June 2015). No pathogenic variants in these alternate genes were found.

\section{DISCUSSION}

Our goal was to evaluate the effect of heterozygous deleterious variants in MYO1A on sensorineural hearing using the genotypic ascertainment approach to clinical research. By leveraging the ClinSeq resource, we were able to identify a cohort of individuals with rare and predicted deleterious variants in MYO1A for post hoc evaluation of their hearing acuity. This approach to genotype-phenotype correlation reduces the ascertainment bias of the conventional phenotypic ascertainment approach.

None of our study subjects with MYO1A variants had a hearing loss phenotype resembling that was originally claimed ${ }^{2}$ to be associated with variants in this gene. Although Donaudy et al did not publish audiograms, the hearing losses were described as sensorineural, mostly moderate to profound with variable age of onset from congenital to late onset. Individual patients were described as having bilateral severe hearing loss p.(Val306Met) and p.(Ser910Pro); moderate to severe bilateral sensorineural hearing loss p. $\left(\operatorname{Arg} 93^{*}\right), c .349-350$ insCTT, and p.(Gly674Asp); moderate to severe unilateral sensorineural hearing loss with early onset p.(Glu385Asp); moderate sensorineural hearing loss p.(Ser797Phe); and mild sensorineural hearing loss p.(Gly662Glu). Our dataset includes five individuals with the same mutations reported to be pathogenic in the original report ${ }^{2}$ and seven individuals with predicted pathogenic variants, including two putative null variants. Two individuals did have hearing worse than the 95th centile of the ISO norms, and both were very mild low-frequency losses, which is not considered to be clinically significant hearing loss (Supplementary Table 2). Our data cannot exclude the hypothesis that these MYO1A variants are genetic modifiers that increase penetrance, instead of acting as primary disease-causing variants. The existence of such modifiers has been suggested for other disorders, such as hereditary breast and ovarian cancer. ${ }^{14}$ Alternative hypotheses could include that MYO1A variants predispose to a peculiar sensitivity to environmental noise or pharmacologic toxicity, or that penetrance is dependent upon interaction with variants in other genes. ${ }^{15,16}$ Future studies to evaluate this hypothesis, such as interactome analyzes, could be fruitful. 
Other studies have failed to support the original MYO1A ADNSHL report. $^{2}$ Although Myola is expressed in the mouse cochlea, it is not essential for hearing. ${ }^{4}$ In another study, deafness-associated genes were evaluated in a cohort of 109 hearing-impaired individuals. ${ }^{3}$ Three individuals had variants in MYO1A, including c. $2220 \mathrm{~T}>\mathrm{G}$ p. $\left(\mathrm{Tyr} 740^{\star}\right)$, c.784T $>\mathrm{C}$ p. $\left(\operatorname{Arg} 262^{\star}\right)$, and c.1985G $>\mathrm{A}$ p. (Gly662Glu), but none of the variants co-segregated with hearing loss in the pedigrees. Additionally, p.(Arg262*) was homozygous in one patient with normal hearing.

In summary, we have employed a genotype ascertainment study design that refutes the conclusion that mutations in MYO1A cause ADNSHL. When combined with published mouse data and other human data that fail to support this hypothesis, there are now more studies arguing against pathogenicity, as opposed to the single initial study in favor of pathogenicity. Our research groups have removed MYO1A from the list of genes associated with ADNSHL and we encourage others to consider doing the same.

\section{CONFLICT OF INTEREST}

LGB receives royalties from Genentech and honoraria from WileyBlackwell and is an uncompensated advisor to the Illumina Corp. AJG holds U.S. patents: 7166433 (Transductin-2 and Applications to Hereditary Deafness), 7192705 (Transductin-1 and Applications to Hereditary Deafness), and 7659115 (Nucleic Acid Encoding Human Transductin-1 Polypeptide) and has a pending licensing agreement with EMD/Millipore for anti-TMC antibodies.

\section{ACKNOWLEDGEMENTS}

The authors are grateful to the ClinSeq participants for their contribution to this work. All authors were supported by the Intramural Research Program of the National Institutes of Health, either the National Human Genome Research Institute (LGB, JP, and JJJ) or the National Institute for Deafness and Communicative Disorders (CB, WC, and AJG).
1 Morton NE: Genetic epidemiology of hearing impairment. Ann N Y Acad Sci 1991; 630: 16-31.

2 Donaudy F, Ferrara A, Esposito L et al: Multiple mutations of MYO1A, a cochlearexpressed gene, in sensorineural hearing loss. Am J Hum Genet 2003; 72: 1571-1577.

3 Eisenberger T, Di Donato N, Baig SM et al: Targeted and genomewide NGS data disqualify mutations in MYO1A, the "DFNA48 gene", as a cause of deafness. Hum Mutat 2014; 35: 565-570.

4 Tyska MJ, Mackey AT, Huang JD, Copeland NG, Jenkins NA, Mooseker MS: Myosin-1a is critical for normal brush border structure and composition. Mol Biol Cell 2005; 16: 2443-2457.

5 MacArthur DG, Manolio TA, Dimmock DP et al: Guidelines for investigating causality of sequence variants in human disease. Nature 2014; 508: 469-476.

6 Facio FM, Sapp JC, Linn A, Biesecker LG: Approaches to informed consent for hypothesis-testing and hypothesis-generating clinical genomics research. BMC Med Genomics 2012; 5: 45.

7 Biesecker LG: Hypothesis-generating research and predictive medicine. Genome Res 2013; 23: 1051-1053.

8 Biesecker LG, Mullikin JC, Facio FM et al: The ClinSeq Project: piloting large-scale genome sequencing for research in genomic medicine. Genome Res 2009; 19: 1665-1674.

9 Johnston JJ, Lewis KL, Ng D et al: Individualized iterative phenotyping for genome-wide analysis of loss-of-function mutations. Am J Hum Genet 2015; 96: 913-925.

10 Johnston JJ, Teer JK, Cherukuri PF et al: Massively parallel sequencing of exons on the $\mathrm{X}$ chromosome identifies RBM10 as the gene that causes a syndromic form of cleft palate. Am J Hum Genet 2010; 86: 743-748.

11 Kircher M, Witten DM, Jain P, O'Roak BJ, Cooper GM, Shendure J: A general framework for estimating the relative pathogenicity of human genetic variants. Nat Genet 2014; 46: 310-315.

12 International Standards Organization: ISO-7029:2000. Acoustics-statistical distribution of hearing thresholds as a function of age. Geneva 2000.

13 Mazzoli M, Van Kamp G, Newton V, Declau G, Parving A: Recommendations for the description of genetic and audiological data for families with nonsyndromic hereditary hearing impairment. Audiol Med 2003; 1: 148-150.

14 Evans DG, Shenton A, Woodward E, Lalloo F, Howell A, Maher ER: Penetrance estimates for BRCA1 and BRCA2 based on genetic testing in a clinical cancer genetics service setting: risks of breast/ovarian cancer quoted should reflect the cancer burden in the family. BMC Cancer 2008; 8: 155.

15 Riazuddin S, Castelein CM, Ahmed ZM et al: Dominant modifier DFNM1 suppresses recessive deafness DFNB26. Nat Genet 2000; 26: 431-434.

16 Schultz JM, Yang Y, Caride AJ et al: Modification of human hearing loss by plasma-membrane calcium pump PMCA2. N Engl J Med 2005; 352: $1557-1564$.

Supplementary Information accompanies this paper on European Journal of Human Genetics website (http://www.nature.com/ejhg) 